\title{
DOCUMENTATION
}

\section{Les grands Transports d'Energie électrique à haute tension}

\author{
On pose en Savoie, sur la ligne Moûtiers-Lyon, un câble souterrain isolé à 150.000 volts, \\ courant continu
}

\author{
par V. Sruvestre, Ingénicur A. M. el $I . E . G$.
}

\begin{abstract}
La ligne de transport d'énergie de Moûtiers à Lyon a été exécutée et mise en service en 1906. Son but, à l'origine, était essentiellement d'assurer le service des tramways de la Compagnie des Omnibus et Tramways de Lyon, et l'on a adopté pour ce transport d'énergie le courant continu à haute tension à intensité constante et tension variable dit système "Thury ". Cette première ligne réunissait l'usine génératrice de Plombière-Saint-Marcel, près Moûtiers (Savoie), aux usines réceptrices de Vaulx-en-Velin (Rhône), et de la rue d'Alsace, à Lyon. Dans ce système spécial de transport d'énergie, les machines génératrices et réceptrices sont toutes connectées en série; l'intensité maintenue constante est réglée à l'usine génératrice en faisant varier la vitesse des turbines hydrauliques. La vitesse des réceptrices est réglée automatiquement par des régulateurs de vitesse agissant par décalage des balais.
\end{abstract}

Les principales caractéristiques de ce premier transport sont :

Longueur ................. $178 \mathrm{~km}$.

Puissance transportée .............. $3.000 \mathrm{kw}$.

Tension au départ............... 57.600 volts

Intensité constante.............

Entre Vaulx-en-Velin et la station de la rue d'Alsace à Lyon, existe un câble souterrain de 5 kilomètres de longueur, $75 \mathrm{~m} / \mathrm{m}^{2}$ de section, établi pour une tension de service de 50.000 volts.

La station réceptrice de la rue d'Alsace est équipée avec des groupes moteurs série-génératrices, à courant continu 600 volts. Ces groupes transforment donc le courant continu à tension variable et intensité constante, en courant continu à tension constante.

La station de Vaulx-en-Velin est équipée exclusivement avec des groupes moteurs série-alternateurs triphasés. Cette disposition très originale a été admise afin de donner, dès l'origine, aux différents services de la Société Générale de Force et Lumière, le maximum de souplesse possible, sans avoir pour cela des réseaux intimement liés. En effet, avec des groupes ainsi constitués, il est possible de renforcer les réseaux généraux triphasés des départements de l'Isère, du Rhône et de la Drôme, par le transport série Moutiers-Lyon à courant continu, et, inversement, de recevoir de ces réseaux triphasés du courant que l'on peut transformer, par les alternateurs marchant en moteurs synchrones et les moteur ssérie marchant en génératrices, en courant continu série à intensité constante.

M. Boissonnas a déclaré, au dernier Congrès de la Houille Blanche, que cette distribution s'est montrée en pratique excellente et qu'on avait considérablement augmenté sa puissance.

Tout d'abord l'intensité d'origine de 75 ampères a été portée dès 1910 à 150 ampères, et les nouvelles usines génératrices de Bozel, sur la Rozière, en Savoie $(9.000 \mathrm{KW}$.) et de Fonds-deFrance, sur le Bréda en Isère $(6.000 \mathrm{KW}$., lacs des Sept-Laux), sont venues renforcer l'usine de la Plombière, et porter la capacité pratique du transport d'énergie de Moûtiers-Lyon à 15.000 kilowatts à la tension normale de 100.000 volts. Cette ligne était encore, ces dernières années, celle fonctionnant en France à la tension la plus élevée; elle n'a été dépassée que par les transports en courant triphasé 120.000 volts de nos grandes lignes d'interconnexion et 150.000 volts de la Compagnie des Chemins de fer du Midi. Elle est à notre connaissance actuellement unicue en France dans son genre.

Depuis 1906, des progrès remarquables ont été réslisés dams la construction des génciratrices à courant continu à haute tension: à cette époque, la tension maximum par collecteur ne clépassait pas 3.600 volts. En 1910 , cette tension atteignait 4.500 volts, et cette année, on remarquait, à l'Exposition de la Houille Blanche, un groupe triple de 150 ampères, pouvant donner directement 7.200 volts par collecteur, soit une tension totale directe de 21.600 volts pour le groupe.

Au point de vue service, ce transport d'énergie à courant continu est bien équilibré : en effet, l'usine de la Plombière qui prend l'énergie au fil de l'eau, constitue en quelque sorte le fond du transport, celui destiné à assurer les pertes ; l'usine de Bozel est complétée par un réservoir journalier transformant l'énergie de mit 'n énergie de jour et enfin l'usine de Fonds-de-France, utilisant l'eau des lacs des Sept-Laux (cote 2.130 mètres), dispose d'un réservoir saisonnier remarquable puisqu'il permet d'emmagasiner, pour être utilisés l'hiver, 9.000.000 de mètres cubes sous une chute brute de 1.032 mètres. C"est la plus haute chute de Franee ave" celle du Bàton, des Etablissements Keller \& Leleux.

La partie la plus accidentée de cette ligne est la traverséc de la montagne du Chat, entre Chambéry et Novalaise, par le col de Lépine (altitude 1.003 mètres), sur un parcours d'environ $30 \mathrm{~km}$.

En vue d'augmenter la sécurité d'exploitation de la ligne, ct d'en supprimer le plus possible l'entretien dans la partie montagneuse, où les intempéries la rendaient, surtout en hiver, difficile d'accès, la Société Générale de Force et Lumière s'est décidée à faire passer cette ligne en souterrain, à l'aide d'un câble armé à très haute tension.

En prévision de l'augmentation de puissance de ce transport d'énergie, le câble souterrain Chambéry-Novalaise a été prévu pour la tension de service de 150.000 volts par rapport à la terre, et pourra atteindre 300.000 volts entre fils positif et négatif, le point milieu étant mis à la terre, valeur tout à fait remarquable qui marquera une date dans la construction des câbles. On sait. ell effet, qu'industriellement, on n'a pas encore dépassé les tensions de 60 à 70.000 volts pour les câbles à courant alternatif : l'Union d'Electricité, à Paris, possède environ $400 \mathrm{~km}$. de câble à 60.000 volts, ce qui est la plus haute tension en France pour des câbles à courants alternatifs. On a effectué dernièrement en Italie la pose d'un câble à 70.000 volts alternatif, et les techniciens ont heaucoup remarqué à l'Exposition de la Houille Blanche un échantillon de câble isolé au papier imprégné qui fait l'objet d'essais à 130.000 volts alternatif dans les laboratoires du constructeur.

La fabrication clu câble : Chambéry-Novalaise a nécessité des précautions toutes spéciales, eu égard à l'importance de la tension sous laquelle il doit fonctionner.

Comme le disait récemment un ingénicur de la Compagnie Générale des Câbles qui a suivi sa fabrication, on sait que l'isolement des câbles est à l'heure actuelle assuré uniquement par du papier imprégné, papier spécial découpé en bandes que l'on en- 
roule sur les conducteurs en épaisseur variable suivant la destinatıon du câble que l'on rlessèche ensuite et que l'on imprègne avec de la matière isolante pâteuse avant de recouvrir le tout d'un tube de plomb uniquement destiné à protéger l'isolant contre l'humidité.

Les ennemis du bon isolement d'un câble, sont en effet : l'hu' midité et l'air, qu'il faut l'un et l'autre extraire complètement du câble pour les remplacer par une matière isolante convenable, en l'espèce, un mélange d'huile de naphte et de résine.

L'enveloppe de plomb est elle-même protégée mécaniquement par une armature de deux fers feuillard enroulés à recouvrement après interposition d'un matelas de filin goudronné.

Dans les parties abruptes du tracé, on a remplacé le fer feuillard par deux couches de fil d'acier fretté de distance en distance, de manière à amarrer chaque longueur de câble dans le rocher.

La pose de ce câble présente une innovation intéressante en ce qui concerne la recherche rapide des défauts; on a prévu à chaque jonction un petit conducteur en cuivre relié à une borne en ciment, permettant la recherche des défauts par la méthode bien connue des chutes de tension.

Enfin, tous les câbles ont été essayés en plate-forme sous une tension continue de 300.000 volts, et, pendant la pose, des essais partiels ont eu lieu aussi fréquemment que possible, sous la tension de 200.000 volts, à l'aide d'un appareil à contact tournant transportable.

Il était intéressant, après l'Exposition de Grenoble, de signaler l'effort et les progrès remarquables réalisés par les constructeurs français de câbles souterrains, et l'esprit de hardiesse de l'exploitant qui, avec une grande persévérance, perfectionne sa ligne à courant continu série "Moûtiers-Lyon ", ligne actuellement unique au monde et dont la tension atteindra probablement un jour la valeur de 300.000 volts, n'ayant rien à envier à celles employées (il est vrai en courant alternatif) sur les super-réseaux américains.

V. Sylvestre.

\section{Les extensions des centrales thermiques aux Etats-Unis}

D'importantes modifications et extensions viennent d'être exécutées ces temps derniers, en de nombreuses centrales des Etats-Unis, parmi lesquelles certaines présentent des caractéristiques vraiment nouvelles et intéressantes. Il y a lieu de mentionner, en particulier, dans l'usine de "Centrale River " de la "Bulfalo G. E. Co " un groupe avec turbine à vapeur de $60.000 \mathrm{KW}$. travaillant à 15 kilos et sous $120^{\circ}$ de surchauffe, accouplé avec un alternateur de $66.667 \mathrm{KVA} . \div 12.000$ V. $\div 25$ p. p. s. $(\cos \approx=0,90)$ tournant à 1.500 t. m. ; et, dans la centrale de "Trenton Channel, 2 turbo-alternateurs de $50.000 \mathrm{KW}$. turbine à 25 kilos $\div 1.200 \mathrm{t} . \mathrm{m}$. ; alternateur de $62.500 \mathrm{KVA} .12 .200 \mathrm{~V} . \div 60 \mathrm{p}$. s.

Elettrotechnica (cron.), 25 mai 1926.

\section{La commande électrique des vannes sur les conduites de vapeur}

Cette commande vient d'être appliquée dans la centrale thermique de Samerset (U. S. A. Mass.) pour les vannes de retenue et de prise des chaudières, pour celles de sectionnement, sur les conduites principales de 18 pouces et pour la vanne principale sur la tuyauterie d'admission des turbines.

Les vannes sont commandées au moyen de petits moteurs $C$. $C$. à 90 ou $140 \mathrm{~V}$. alimentés par la batterie d'accus de réserve de la centrale. Les commandes sont réunies sur un tableau unique, ainsi que les signalisations lumineuses de la manœuvre à distance avec celles des vannes d'admission de l'huile combustible dans les réservoirs et celles de l'eau de circulation.

Elettrotecnica (cron.), 25 mai.

\section{Dispositif pour la mesure électrique des petites pressions des gaz}

Le dispositif décrit en cet article utilise un circuit oscillant avec ion à 3 électrodes dans lequel l'inductance d'une bobine varie selon qu'on approche ou éloigne d'elle une petite lame métallique mue par un léger diaphragme de mica qui ferme le réservoir à gaz. Un micro-ampéremètre inséré dans le circuit anodique donne un déviation de $1 \mathrm{c} / \mathrm{m}$ pour un courant de $10^{8} \mathrm{~A}$. qui correspond à une variation de pression dans le réservoir à gaz, égale à $7 \times 10^{6}$ centimètres de colonne d'eau.

Engineer, 22 janvier 1926.

\section{Le plus grand monte-charge du monde}

Il semble bien qu'il vient d'ètre installé dans une mine sudafricaine pour assurer le service de l'exploitation dans un puits de 1.500 mètres de profondeur. Cet appareil comporte 2 tambours cylindro-coniques présentant aux extrémités des diamètres respectifs de 5 mètres et 12 mètres et auxquels sont directement accou- plés 2 moteurs électriques de $1.800 \mathrm{KW}$ chacun, capables de lui imprimer une vitesse de rotation de $33 \mathrm{t}$. $\mathrm{m}$. pour le soulèvement d'une charge de 10 t. L'engin est conçu, en outre, pour pouvoir faire face à des pointes exceptionnelles de $6.750 \mathrm{KW}$.

Elettrotecnica (cron.), 15 juin.
J. B.

\section{Le laboratoire d'essais des turbines hydrauliques de la Maison Bell, à Kriens}

Dans une série de 3 articles consécutifs, l'auteur expose en détail avec plans, dessins et photos à l'appui, la conception de cette installation, dans laquelle 3 pompes Sulzer de divers types, alternativement ou simultanément commandées par un moteur synchrone de $300 \mathrm{CV}$., un réservoir de $143 \mathrm{~m}^{3}$ dont l'eau est déversée dans une bâche d'alimentation et, de là, dans la turbine à essayer.

La station comporte, pour les HP., MP. et BP., 3 dispositifs d'essais munis, chacun, des appareils correspondants de mesures, réglage et sécurité

Schw. Bzg., 27 février, 6-13 mars.

J. B

\section{La protection des appareils de mesure contre les détériorations accidentelles}

Assurer cette protection pour éviter toute détérioration de ces appareils, est aujourd'hui capital, tant en raison de leur multiplicité dans les installations de quelque importance, que du prix, fort élevé, de leurs réparations.

L’anteur de cet article étudie en détail les causes les plus fréquentes des détériorations habituelles (secousses, trépidations, échauffements, surintensités et surtensions) et indique, dans chacun de ces cas, les précautions à prendre dans leur construction comme dans leur emploi. Il donne, en terminant, un intéressant exposé des prescriptions réglementaires en vigueur en Allemagne pour l'exécution des essais aux surtensions.

Zeilsch. des V. D. Ing., 6 février 1926. 


\section{Los moteurs synchrones et asynchrones synchronisés à marche lente}

Certaines industries (mines, frigorifiques, chimiques), utilisant en assez grand nombre des compresseurs d'air, ont besoin pour les actionner électriquement, de moteurs à marche lente, et les constructeurs les plus cotés ont dû orienter une branche de leur fabrication vers les engins susceptibles de satisfaire à cette exigence sans rien sacrifier du fonctionnement, ni du rendement. Ils essayerent d'abord les moteurs asynchrones, mais ceux-ci présentent l'inconvénient d'un facteur de puissance médiocre; ils furent, ensuite, aiguillés vers les moteurs synchrones et asynchrones synchronisés, qui semblent bien, nous dit l'auteur, devoir fournir une solution définitive de la question. Il nous en domne, comme exemples, la description de différents groupes moto-compresseurs en usage courant clans les mines.

R. G. E., 9 janvier.

\section{Conditions de coexistence des transports de force et des lignes de télécommunication}

On comait la gène mutuelle à laquelle conduit cette coexistence. L'auteur se propose de déterminer les conditions dans lesquelles cette gène peut ètre réduite à son minimum, tant en ce qui concerne les perturbations que le danger. En ce qui concerne les lignes téléphoniques à grande distance, à coefficient d'utilisation toujours très élevé, les prescriptions imposéès lui semblent trop draconiennes pour beaucoup de lignes, et, quant aux transports de force, il estime que, pour les tensions ne dépassant pas $60.000 \mathrm{~V}$. quelques prescriptions très simples leur permettraient de coexister avec les précédentes sans grand préjudice pour ces dernières. 1l ajoute, toutefois, qu'avant d'arrèter les valeurs numéricques de traduction de ces prescriptions, de nouvelles expćriences seront nécessaires.

J. B.

Bulletin de la Socióte Francaise des Electriciens. Janvier, 1926.

\section{La traversée des Rios Guadalete et Majaceite par siphons renversés en béton armé}

L'importance et surtout la soudaineté des crus du Rio Guadalete. comme du reste de son affluent, le R. Majaceite (Prov. de Cadix) a conduit à effectuer leur traversée par 2 siphons renversés de $40 \mathrm{~m}$. de portée sur $15 \mathrm{~m}$. de flèche, dans lesquels le métal de ces traversées classiques est remplacé par le B. A., qui tend. de plus en plus, à s'y substituer.
L'auteur de cet intéressant article, après un exposé détaillé lle la construction de cet ouvrage (illustré abondamment de figures et photos) donne le calcul res tores qui a été appliqué en la circonstance.

Schw., Bzg., 27 février 1926.

J. B.

\section{La prévention de l'échauffement dans les barres-omnibus, par leur dimensionnement}

On sait que l'échauffement de régime d'une barre qui transporte un courant constant. s'obtient quand la quantité de chaleur développée, par seconde, égale celle que dissipe la barre par convection et rayonnement, laquelle dépend elle-même du dimensionnement de la barre, ainsi que des conditions atmosphériques. Le calcul de ce dimensionnement est présenté, dans cet article, de façon aussi claire que pratique, accompagné d'abaques qui en rendent l'appli- cation facile.

Toutefois les résultats que fournissent méthode et abaques, daus l'établissement des avant-projets, demandent à ètre corrigés par l'introduction du facteur "ventilation des barres ", essentiellement variable et a une grande importance que, seule, peut étahlir l'expérience.

Jeumont, 10 décembre.

J. 13.

\section{Les installations hydro-électriques automatiques}

Un groupement important d'installations de ce type vient d'être mis en service récemment, sur le fleuve Peshtigo (Wisconsin U.S. A.). Il comporte 3 centrales fournissant ensemble $77.200 \mathrm{KVA}$. et fonctionnant entièrement par automatisme. La mise en marche des groupes est obtenue en utilisant la chute de fréquence.
Les 3 centrales peuvent cependant, ètre eommandées, à distances, de certaines autres usines. Le système s'est révélé parfait, tant comme économies réalisées que comme régularité du service assuré.

Electr. World, 7 février 1926.

\section{Le plus grand turbo-alternateur du monde}

La Société "Brown-Boveri " nous informe qu'elle vient d'obtenir la commande d'un groupe turbo-alternateur de $160.000 \mathrm{KW}$. pour New-York.

Cette commande leur a été transmise par l'Américan BrownBoveri Electric Corporation, pour la Centrale de Hellgate de l'United Electric Light and Power Co, à New-York.

Cet ordre est considéré, à juste titre, par la Société "BrownBoveri ", comme un grand succès, étant donné la concurrence très importante des firmes américaines.

Le choix d'un tel groupe dont la puissance dépasse tout ce qui a été construit dans le monde jusqu'à ce jour, est motivé par les prix énormes du terrain à New-York. Les conditions imposées au constructeur exigeaient, en effet, le placement d'un groupe d'une puissance aussi élevée que possible dans l'espace encore disponible de la centrale de Hellgate.

Le constructeur a proposé un groupe de $160.000 \mathrm{KW}$. turbine à réaction pure constituée par :

Un cylindre à haute pression $1.800 \mathrm{t} / \mathrm{m}$ avec génératrice triphasée de $75.000 \mathrm{KW}$.

Un cylindre à basse pression à extrémités doubles pour $1.200 \mathrm{t} / \mathrm{m}$. avec une génératrice triphasée de $85.000 \mathrm{KW}$., pression $19.6, \mathrm{~kg}$. : $\mathrm{cm}^{3}$. Les génératrices seront bobinées pour $13.800 \mathrm{~V} \cos 0,85$, fréquence $60 \mathrm{Ps}$.
Caractéristiques générales.

\section{a) Turbine}

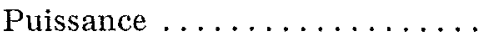

Nombre de tours............

Poids du cylindre...........

Poids du rotor ..............

Diamètre moyen du rotor .....

Poids total de la turbine complète y compris la plaque de base et les paliers..........

b) Génératrice.

Puids du stator . ............

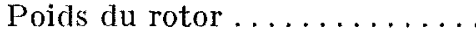

Poids total de la génératrice...

$\begin{array}{cc}\text { Partie haute } & \text { Partie basse } \\ \text { pression } & \text { pression } \\ 75.000 & 85.000 \\ 1.800 & 1.200 \\ 48 \mathrm{t} . & 335 \mathrm{t.} \\ 23 \mathrm{t} . & 87 \mathrm{t} . \\ 1.800 \mathrm{~m} / \mathrm{m} . & 3.900 \mathrm{~m} / \mathrm{m} .\end{array}$

environ $705 \mathrm{t}$

\section{Encombremenl de tout le groupe.}

Longueur : $20 \mathrm{~m} .50$; largeur : $12 \mathrm{~m}$.

Nola. - Les parties les plus lourdes seront construites en Amérique, telles que le cylindre à basse pression, le rotor de la génératrice à marche lente el les slators des deux génératrices. 


\section{La plus grande conduite en bois}

Il semble bien (que ce soit celle de l'installation H. E. de Copco $n^{\circ}$ 2, sur le Klamalh (Californie du Nord), $30.000 \mathrm{KW}$, qui, sur une longueur de $450 \mathrm{~m}$, présente un diamètre de $4 \mathrm{~m} .85$.

Extérieurement, la conduite est cerclée de fer et est appuyée sur des consoles également en fer, encastrées dans des fondations en béton armé. Elle part de la prise (à la sortie et jonction de 2 galeries) pour aboutir à la chambre de mise en charge, d'où partent les conduites en acier aboutissant à la centrale sous $48 \mathrm{~m}$.

15 mars 1926.

J. B.

\section{Commande à distance des sous-stations}

Ayant besoin d'accroître sa capacité, dans le courant de l'année dernière, la sous-station de Jamaïca (New-York) fut conduite, après étude serrée des 3 solutions qui s'offraient (augmenter son importance actuelle, construire une nouvelle station semblable ou construire une sous-station de moindre importance fonctionnant sans opérateur, automatiquement et à distance), à admettre la $3^{e}$ de ces solutions qu'elle réalisa à Woodhaven ( $4 \mathrm{~km}$. de Jamaïca) sous la forme d'une $1^{\text {re }}$ sous-station (d'autres étant prévues pour l'avenir) comportant 3 transfos triphasés auto-réfrigérants dans l'huile, de $3.750 \mathrm{KVA}$, dont 1 en réserve, alimentés, souterrainement, par un câble à $13.200 \mathrm{~V}$.

Journal of the A. I. E. E., juin 1926.

\section{Les nouveaux aménagements hydrauliques en Georgie (U. S. A.)}

Depuis qu'elle a pris en main l'exploitation des ressources hyodrauliques de l'Etat de Géorgie, la "Georgia Railway and Power (o " poursuil d'importants aménagements, spécialement sur les rivières Tugalo et Tallulah. La tâche était ardue, car la connaissance de leur régime était imparfaite; mais, depuis 1916, une documentation sérieuse a été réunie qui a permis d'étudier, néanmoins, assez rationnellement, leur régularisation (sur laquelle l'article donne d'abondants détails) et de mettre en service 4 cen trales : Tallulah $(72.000 \mathrm{KW}$ ) ; Tugalo $(50.000)$; Yonah et Terrora $\left(40.000 \mathrm{KW}\right.$.) ; cependant que la $5^{\mathrm{e}}$, en cours d'achèvement, fournit déjà $25.000 \mathrm{KW}$. à Nacoochee. La mise en service de la $6^{\mathrm{e}}$ portera à $181.000 \mathrm{KW}$. la puissance totale disponible pour la Société.

Electr. World, 10 avril 1926.

\section{La sous-station automatique de Bourbon}

Cette sous-station transforme du triphasé $6.600 \mathrm{~V}$. en continu 200130 V. (3 conductrices 3 feeders tripli $6.600-60$ p. s. ; elle alimente 2 groupes moteurs-générateurs de 1.000 et $1.200 \mathrm{KW}$. Le $1^{\text {ar }}$ groupe comporte un moteur synchrone de $1.350 \mathrm{KVA}$. tournant à 900 t. m. entrainant la génératrice continue $260 \mathrm{~V}$. en même temps qu'une excitatrice de $15 \mathrm{KW}$. $-125 \mathrm{~V}$. Le $2^{\mathrm{e}}$, tournant à 100 t. m.. actionne la génératrice à $300 \mathrm{~V}$. et l'excitatrice de $3 \mathrm{KW} .-75 \mathrm{~V}$.
Le démarrage, comme l'arrêt, sont automatiques et, en outre, un système est prévu, qui assure une protection absolue dans 19 cas de fonctionnement anormal. En cas de court-circuit dans le réseau, le disjoncteur agit sur le champ différentiel série.

Avec pointes atteignant jusqu'à $2.400 \mathrm{KW}$. à certaines heures, la puissance moyenne fournie par cette sous-station est d'environ $30.000 \mathrm{KWh}$. par jour.

Gen. Electr. Review, février 1926.

\section{La nouvelle centrale de Berlin}

L'auteur nous présente ici, en clétails, les dispositions adoptées pour la nouvelle contrale municipale de Berlin, et dont certaines présentent grand intérèt. Initialement, elle comprendra 3 unités de $70.000 \mathrm{KW}$. chacune fournissant des triphasés à $6.000 \mathrm{~V}$., transformés en 30.000 pour le transport, 16 chaudières, de types variés, mais présentant chacune 1.750 mètres carrés de surface, timbrées à $33 \mathrm{~kg}$, et groupées par 2 dans une cheminée de $70 \mathrm{~m}$., à tirage aspiré, fourniront la vapeur par combustion de charbon pulvérisé et séché dans une installation située près de la chaufferie. Comparant les caractéristiques de cette nouvelle centrale avec celles de Golpa, construite pendant la guerre (1916), il souligne les progrès réalisés principalement dans le timbre - qui passe de 16 à 33 kilos - et dans la surface de chauffe unitaire, qui passe de 500 à 1.750 mètres carrés.

Chaleur et Industrie, avril 1926.

J. B.

\section{La chute et la distribution d'eau de l'Alto-Timavo}

En vue d'alimenter Trieste en eau potable, la Société Idroelettrica de l'Alto Timavo dut concevoir et réaliser un important projet d'aménagement du bassin du Timavo et du Recca, qui lui permettra de disposer prochainement de 180 millions de KWh par an aux portes de la ville. Les rivières Padez et Suhorca fournissent à l'adduction d'eau ; la production d'énergie est demandée au Recca et au Tamavo, régularisés en raison de la grande variation de leurs débits qui, de $13 \mathrm{~m}^{3} / \mathrm{sec}$ peut descendre à $1 \mathrm{~m}^{3}$.

La centrale comporte 5 groupes Pelton, de chacun 15.000 CV., fonctionnant, sous-station $330 \mathrm{~m}$. de chute, avec l'eau de ces 2 torrents. De son côté, l'eau du Padez permet d'actionner 3 groupes de $1.000 \mathrm{CV}$. chacun, fournissant un appoint de $3.000 \mathrm{CV}$. à l'installation principale.

Une installation de stérilisation et filtrage des eaux d'alimentation de la ville, capable de $50.000 \mathrm{~m}^{3} /$ jour (susceptible d'ètre portés bientôt à 90.000 ) est annexée à l'aménagement.

Sincronizzando, mai 1926.

J. B.

\section{La chute de la Camarasa}

Poursuivant ses aménagements du bassin de l'Ebre, la Société Riegose fuerzas del Ebro vient d'équiper récemment une nouvelle chute de $100.000 \mathrm{CV}$. à proximité du confluent de la Segre et de la Noguera Pallaresa (Catalogne). Cette chute comporte d'importants ouvrages, en particulier le barrage accumulateur, un des plus hauts d'Europe $(102 \mathrm{~m}$.), retenant 160 millions de mètres cubes, et les déversoirs automatiques à secteurs en béton armé, de $10 \mathrm{~m}$. de rayon, qui s'effacent entièrement dans des logements excavés, en roche compacte pour l'évacuation des crues. 210.500 metres cubes de béton ont été mis en ouvre pour ces ouvrages.
L'usine génératrice est accolée à une paroi rocheuse abrupte et, par partie, logée dans la roche même. Après achèvement, elle comportera 5 groupes turbo-alternateurs - dont 2 sont déjà installés - comprenant chacun une turbine Francis, développant $18.500 \mathrm{CV}$. à $375 \mathrm{t}$. m., sous $82 \mathrm{~m}$. de chute, accouplée à 1 alternateur G. E. C. dont le pivot supérieur porte l'inducteur et le rotor de la turbine.

Le courant produit est du triphasé $6.600 \mathrm{~V}$., qui est transporté à Barcelone apris élévation à $110.000 \mathrm{~V}$.

Genie Givil, 15 mai 1926. 


\section{INFORMATIONS}

\section{Union des Syndicats de l'Electricité}

25, Boulevard Malesherbes, Paris

Téléphone : Elysées 31-82.

Essais contrólés de moteurs électriques portatifs à usages agricoles. organisés en janvier 1927 par l'Union des Syndicats de l'électricité, boulevard Malesherbes, 25, à Paris, avec le concours du Ministère de l'Agriculture, de la Fédération Nationale des Collectivités d'Electrification Rurale et de la Chambre Syndicale des Constructeurs de Machines Agricoles de France.

NOTE. - Etant donné le développement rapide des applieations agricoles de l'électricité, il est devenu évident qu'il ne faut laisser évoluer au hasard ni les moteurs électriques destinés à l'agriculture, ni les appareils agricoles que ces moteurs doivent actionner.

De multiples questions se posent à l'heure actuelle à ce sujet : $\mathrm{i}^{\mathrm{l}}$ faut faciliter à l'agriculteur le choix des moteurs qu'il doit employer et l'adaptation de ces moteurs aux outils agricoles, il faut perfectionner les transmissions et améliorer le rendement de l'ensemble mécanique que forment le moteur et l'outil, enfin il faut normaliser le plus possible les moteurs, les outils et les éléments constitutifs des uns et des autres, afin de réduire leurs prix de revient et de faciliter à l'agriculteur l'approvisionnement des pièces de rechange.

La simple énumération de ces besoins constitue un véritable programme : il s'agit en un mot d'adapter l'emploi de l'électricité aux usages agricoles et de réaliser l'entente des constructeurs de moteurs électriques avec les constructeurs d'outils agricoles.

En vue de répondre au désir qui lui a été exprimé par le Ministère de l'Agriculture, l'Union des Syndicats de l'Electricité a constitué une commission composée de représentants du Corps du Génie Rural, de la Chambre Syndicale des Constructeurs de Machines Agricoles de France, de la Fédération Nationale des Collectivités d'Electrification Rurale et enfin des divers Syndicats qui la composent, notamment des syndicats de distributeurs d'énergie électrique et des syndicats de constructeurs de matériel électrique.

Cette commission fonctionne depuis le mois de mai 1926 sous la présidence de $M$. Drin, l'un des membres les plus compétents de l'Union des Syndicats de l'Electricité.

Elle a pour vice-présidents : MM. Le Couppey de La Forest, inspecteur général du Génie Rural; Gougis, ancien président de la Chambre Syndicale des Constructeurs de Machines Agricoles, et Leroy, délégué des constructeurs de moteurs électriques.

Après avoir tracé le plan de ses travaux, la commission a déciclé, pour aller au plus pressé, de procéder à un inventaire des moteurs et appareils qui existent actuellement en n'envisageant tout l'abord que les moteurs portatifs : il lui semble, en effet, que c'est sur ces appareils qu'il est nécessaire de porter ses premiers efforts.

Mais elle n'a pas voulu se limiter à cet inventaire et elle tient aussi à se faire une idée aussi exacte que possible de la valeur des appareils. Tout en écartant l'idée d'un concours qui conduirait à un classement, elle s'est arrêtée à l'organisation d'essais contrôlés qui permettront d'examiner avec toutes les garanties et toute l'impartialité nécessaire les moteurs qui seront présentés et de préparer ainsi son neuvre ultérieure de normalisation.

Nous donnons ci-dessous le programme de ces essais contrôlés tel qu'il vient d'être arrêté par la commission d'organisation dans sa séance du 5 juillet 1926 .

Nous attirons particulièrement l'attention de tous les constructeurs de moteurs sur l'occasion qui leur est offerte de faire connaitre leurs fabrications et de mettre en évidence les qualités qu'ils ont cherché à réaliser : les moteurs qui prendront part aux essais contròlés seront ensuite exposés au Salon de la Machine Agricole et au Concours Général Agricole afin de les faire connaitre le jlus largement possible à toutes les personnes qui seront appelées à les utiliser.

On remarquera que ces essais ne sont ouverts qu'aux seuls constructeurs français et qu'aux seuls moteurs qui, clans toutes leurs parties, sont exclusivement français.

Pour tous renseignements, prière de s'adresser à l'union des Syndicats de l'Electricité, 25, boulevard Malesherbes, à Paris. (Téléph. : Elysées 31-82 et (04-17).

Des exemplaires du programme seront envoyés à premiere demande et par retour du courrier.

\section{REGILEMEN'T}

Arrèté le 5 juillet 1926 .

Article premier. - L'Union des Syndicats de l'Electriciti, avec le concours du Ministère de l'Agriculture, de la Fédération Nationale des Collectivités d'Electrification Rurale et de la Cham. bre Syndicale des Constructeurs de Machines Agricoles de France, organise des essais contrôlés de moteurs électriques portatifs à usages agricoles.

Ces essais, réservés aux seuls constructeurs français, auront licu

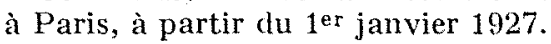

Art. 2. - Objel des essais. - Les essais ont pour objet de mettre en valeur les qualités des moteurs électriques portatifs destinés à actionner les différentes machines en usage clans les exploitations agricoles.

Les points suivants retiendront notamment l'attention du jury :

- Rapidité de mise en action :

- Rendement global mesuré sur l'arbre de la machine conmandée (moteur et réducteur) ;

-- Sécurité du personnel et du matériel;

- Facilité de transport;

- Facilité de substitution clu moteur ;

- Robustesse :

-_ Prix.

AnT. 3. - Calégories. - Les moteurs présentés pourront ètre monophasés ou triphasés et devront être de l'un des trois types suivants :
$1^{\text {re }}$ catégorie : 1,5 ch. $(1,1$ kilowatt $)$
$2^{\text {e }}$ catégorie : 3 ch. $(2,2 \quad-)$
$3^{\mathrm{e}}$ catégorie : 5 ch. $(3,7 \quad \ldots)$

La puissance sera mesurée sur l'arbre du moteur.

Le régime sera celui de la puissance nominale, maintenue pendant une heure.

Chaque participant ne pourra présenter, clans chaque catégorie, plus de deux moteurs de même nature.

An't. 4. - Construction. -- Les moteurs devront ètre de construction exclusivement française et être construits et essayés conformément aux règles de l'Union des Syndicats de l'Electricité.

Ils devront être présentés avec leur câble souple de raccorlement, qui fera partie de l'ensemble.

Ant. 5. - Vilesse de rolation. -- Tous les moteurs devront ètre prévus pour tourner à 1.500 tours par minute à vide.

I.es moteurs triphasés devront fonctionner sous la tension normale de 200 ou 400 volts entre phases. 
Les moteurs à courant continu et les moteurs monophasés devront fonctionner soit à 115 , soit à 230 volts.

ArT. 6. - Réducteurs ou multiplicateurs de vitesse. Disposilif d'entraînemenl. - I c système réducteur ou multiplicateur de vitesse est laissé au choix du constructeur ainsi que le dispositif d'entraînement.

Les appareils devront permettre d'obtenir, sur l'arbre de la machine d'utilisation, les vitesses de $1.500,300,150$ et 50 tours par minute, avec une tolérance de $10 \%$ en moins, et d'entrainer les appareils tournant dans fo sens des aiguilles d'une montre lorsque l'opérateur leur fait face.

I.es vitesses de 3.000 et 750 tours sont facultatives.

AnT. 7. - Support du moteur. - Le mode de fixation du moteur est également laissé à l'initiative du constructeur qui pourra adopter telle disposition qui lui conviendra : moteur supporté par un berceau, sur brouctte (à demeure ou non), etc...

ART. 8. - Machines entrainées. - Les machines agricoles entraînées par les moteurs, s'il en est présenté, devront être de fabrication française, de types normaux.

Celles qui seront entrainées par des moteurs de la première catégorie devront pouvoir, par une transformation simple, être mues à la main.

Ant. 9. - Dépense d'énergie. - La dépense d'énergie électrique $s$ era supportée par les participants.

ArT. 10. - Responsabilités. - L'Union des Syndicats de l'Electricité clécline toutes responsabilités, de quelque nature qu'elles soient, étant entendu que celles-ci incombent aux participants ayant engagé leurs moteurs.

Ant. 11. - Annexes. - Des annexes au présent règlement détermineront ultérieurement les conditions de détail des épreuves auxquelles seront soumis les moteurs.

Aкт. 12. - Jury. - La composition du jury sera déterminée ultérieurement. Il comprendra des représentants du Ministère de l'Agriculture, de l'Union des Syndicats de l'Electricité, de la Fédération Nationale des Collectivités d'Electrification Rurale, de la Chambre Syndicale des Constructeurs de Machines Agricoles de France, de l'Académie d'Agriculture et de la Société des Agriculteurs de France.

Aucun participant aux essais ne pourra en faire partie.

Les décisions du jury seront sans appel.

Ant. 13. - Publication des résultats. - Aucune communication officielle relative aux essais et en particulier aux résultats obtenus, ne pourra être faite que par l'Union des Syndicats de l'Electricité, ou avec son autorisation.
ART. 14. - Rapport général. - Le rapport général des résultats sera présenté en trois chapitres :

a) Règlement et organisation des essais ;

b) Monographies des moteurs engagés;

c) Résultats des essais.

Arт. 15. - Inscription el droits d'engagement. - Les constructeurs désirant participer aux essais devront être français et se faire inscrire à l'Union des Syndicats de l'Electricité, boulevard Malesherbes, 25, à Paris, avant le $1^{\text {er }}$ novembre 1926, en indiquant le nombre, la nature et l'encombrement des moteurs qu'ils présenteront.

Du fait de leur inscription à l'épreuve, les participants s'engagent à observer le présent règlement.

Un droit d'engagement, destiné à couvrir les frais des essais, sera versé par les participants au moment de leur inscription.

Ce droit sera, par moteur, de :

150 francs pour la $1^{\text {re }}$ catégorie.

- 250 - 2e. - -

$350-2-3^{\mathrm{e}}-$

Les sommes ainsi versées resteront acquises à l'Union des Syndicats de l'Electricité alors même que le moteur ne serait pas présenté à l'épreuve, pour quelque cause que ce soit.

Art. 16. - Formule d'engagement. - Dans la formule d'engagement qu'ils signeront, les participants devront spécifier :

- qu'ils sont français et que leurs moteurs sont, dans toutes jeurs parties, de construction exclusivement française ;

- qu'ils acceptent en toutes ses parties le présent règlement et ses annexes;

- qu'ils dégagent les organisateurs de toute responsabilité de quelque nature qu'elle soit, et prennent celle-ci à leur charge ;

- qu'ils se sont assurés à une Compagnie d'assurances notoirement solvable contre tous les accidents causés, soit à eux-mêmes ou à leur matériel, soit à des tiers ou au matériel appartenant à des tiers (la production de la police d'assurance pourra être exigée par les organisateurs);

- qu'ils déclarent accepter toutes décisions de l'Union des Syndicats de l'Electricité ou du jury au sujet de l'application du règlement et s'engagent à ne s'adresser en aucune circonstance aux tribunaux.

L'engagement devra ètre accompagné d'un tableau de renseignements fourni par le participant et conforme à un modèle qui sera établi par l'Union des Syndicats de l'Electricité.

\section{BIBLIOGRAPHIE}

Le jeune Mécano, par Henry de Graffigny. Un volume de 278 pages et 173 figures. Garnier frères, éditeurs, 6, rue des Saints-Pères, à Paris. - En vente à la librairie Rey; Grande-Rue, à Grenoble.

Il est plus que jamais indispensable, à quelque carrière que l'on se destine, d'être industrieux et savoir se servir de ses mains. Non seulement cette habileté rendra les plus grands services dans toutes les circonstances de la vie courante, où il faut savoir se débrouiller, mais elle permettra d'économiser du temps et de l'argent en exćcutant soi-même des objets, meubles, ou appareils. L'auteur, après avoir décrit l'organisation de l'atelier du jeune amateur, les opérations de l'ajustage, comment on travaille le bois, montre comment on peut construire de petites machines à vapeur, des bateaux, des avions, des instruments d'optique, lunettes, puis les sonnettes, téléphones, jusqu'aux postes de T. S. F.

Ce petit livre, très curieux, rendra de grands services aux jeunes gens et amateurs.

(S. V.)
Les Meubles rustiques régionaux de la France. L'Art de reconnaitre les styles, par Emile Bayard, inspecteur de l'enseignement des Beaux-Arts et des Musées. Un volume de 337 pages, avec 229 figures dans le texte. - Garnier frères, éditeurs, Paris. En vente, librairie Rey, Grande-Rue, Grenoble. Prix : 20 francs.

Les diverses régions de la France sont parfumées chacune d'une inspiration personnelle et ornées d'un paysage caractéristique. En dehors de ces émanations, spirituelle et naturelle, il en est une autre, matérielle, celle qui relève de l'éloquence du sol. Or, chacune de ces différences ordonne l'originalité déterminative du folklore français. Cette originalité s'accorde sous les divers ciels, aussi bien avec le geste de l'homme qu'avec les besoins domestiques, dans la maison.

A chaque province, son expression mobilière. A côté du meuble de grand style créé par la. Cour ou le Château, il y a place pour un beau meuble rustique. L'auteur passe en revue les différents styles: 
breton, flamand, normand, alsacien, lorrain, champenois, vendéen, berrichon, auvergnat, bourguignon, savoyard, dauphinois, etc..., le tout illustré par de nombreuses gravures.

(S. V.).

\section{$\star \star \star$}

La dernière loi sur les loyers (loi du ler avril 1926), par Charles Briand, avocat à la Cour d'Appel de Paris. 1 brochure de 10 pages. - Editions du Tableau fiscal, 65, rue de la Victoire. Paris (I.'e). En vente à la librairie Rey, Grande-Rue, Grenoble.

Cette brochure dome le texte intégral de la loi du 1er avril 1926 réglant les rapports des bailleurs et des locataires de locaux d'habitation.

Elle contient 28 pages de commentaire sur le texte de la loi, qui n'est pas moins riche que les précédents en obscurités de toutes sortes, équivoques, amphibologies; voire même en contractictions. Ce document très clair, rendra de grands services, aussi bien aux propriétaires qu'aux locataires.

$$
\text { (S. V.). }
$$

$* *$

Pour comprendre la Mécanique, par l'Abbé Moreux, directeur de l'Observatoire de Bourges. 1 rol. in-16 de 260 pages, avec 157 figures. G. Doin, éditeur, 8 place de l'Odéon, Paris (VIe). - En vente à la librairie Rey, Grande-Rue, à Grenoble. Prix : $12 \mathrm{fr}$.

Ceux qui ont étudié les premiers volumes de cette collection ont pu s'apercevoir qu'il était possible de présenter l'algèbre et la géométrie sous des dehors accessibles aux intelligences les plus réfractaires aux notions positives. L'initiation à la mécanique qu'ils vont aborder maintenant, a été conçue dans le même esprit que les ouvrages précédents.

Elle a été écrite dans le langage de tout le monde et l'auteur en a écarté tout l'attirail des termes rébarbatifs rencontrés en plus d'un volume de ce genre. La mécanique tient à la foìs des sciences mathématiques et des sciences expérimentales; il $\mathrm{y}$ avait donc là une bonne occasion d'appliquer les principes d'algèbre et de géométrie déjà étudiés.

Les jeunes élèves ne rencontreront aucune difficulté sérieuse en cours de route. Fidèle à sa méthode pédagogique, l'Abbé Moreux a su tirer des faits, des conclusions et des principes rigoureux susceptibles de nombreuses applications pratiques. Qu'il s'agisse dis leviers, des machines, de l'énergie, l'élève trouvera toujours une ample moisson de problèmes gradués, destinés à rendre pratiques ses acquisitions dans ce domaine particulier de la science.

C'est probablement la première fois que la mécanique est enseignée avec cette clarté et cette simplicité qui font le mérite des ouvrages du savant auteur.

(S. V.).

\section{$\star^{\star \star}$}

L'Union d'Electricité, par H. Brès, ingénieur E. P., brochure inoctavo, tirée sur papier couché et illustrée de photographies', plans et dessins. Editions de la Revue Industrielle, 57, rue PierreCharron, Paris ( $\left.{ }^{\mathrm{e}}\right)$. Prix, dans ses bureaux : 10 franes.

L'Union d'Electricité a mis en marche, il y a quatre ans, cinc groupes alternateurs de $40.000 \mathrm{KW}$. dans la centrale de Gennevilliers; cette Société a ainsi montré la voie à suivre pour produire, au moyen du charbon, le courant électrique à des prix de plus en plus bas et elle a prouvé que l'industrie française peut lutter de pair avec n'importe quelle industrie du monde.

L'Union d'Electricité n'en est pas restée là ; ses dirigeants actifs, tenant à marcher de l'avant dans l'accomplissement de leur programme, ont complété la supercentrale de Gennevilliers par l'installation de nouveaux groupes unitaires de $50.000 \mathrm{KW}$., dont la puissance dépasse de $25 \%$ ceux qui avaient amené la visite d'ingénieurs du monde entier. D'autre part, elle a réalisé l'extension et le renforcement de la centrale de Vitry où, entre autres, a été installée une chaufferie à charbon pulvérisé comportant des chaudières dont la production horairz peut atteindre 75 tonnes; ce sont, croyons-nous, les chaudières à charbon pulvérisé de plus forte production existant en France.
Nous ne doutons pas que ce ne soit qu'une étape pour l'Union d'Electricité, qui ne cesse de déployer tous ses efforts pour se tenir en tète des progrès de l'industrie, non seulement en ce qui concerne les machines et chaufferies, mais aussi pour tous les autres appareils relatifs à la production et à la distribution de l'énergie.

On trouvera dans la brochure de M. Brès la descriplion des nouvelles installations de l'U. D. E. (groupes générateurs, extension de l'Usine de Vitry, réseaux de càbles, lignes aériennes, sousstations). Cette brochure constitue le complément et la mise à jour de l'ouvrage publié en juin 1922 par M. E. Mercier, administrateur délégué de l'U. D. E. sur l'Union d'Electricité et la Centrale de Gennevilliers, document qui se trouve entre les mains de tous cenx qui s'intéressent à l'expansion de l'industrie française et à la production de l'électricité.

\section{$\star^{\star} \star$}

Annuaire 1926 de la Chambre Syndicale des Forces Hydrauliques. - Un volume de 1003 pages, suivi d'une table analytique et du répertoire technique des industries hydro-électriques de 171 pages. En vente à la Chambre syndicale des Forces hydrauliques, 7, rue de Madrid, Paris, et à la librairie Rey, Grande-Ruc, Girenoble.

\section{Cet important ouvrage est divisć en six parties :}

La première partie donne les statuts, la composition du Consejl d'administration et la table alphabétique des membres de la Chambre syndicale.

La deuxième partie donne des renseignements très détaillés sur les établissements et sociétés faisant partie de la Chambre syndicale.

La troisième partic est consacrée à l'Union des Industries mélallurgiques et minières, à l'Union des syndicats de l'électricité ct à la Société hỹdro-technique de France.

La quatrième partie donne des renseignements généraux sur les ministères.

La cincuième partie est consacrée aux statistiques des aménagements des chutes d'eau et des concessions accordées au $1^{\text {er }}$ jallvier 1925 .

Enfin la sixième partie, la plus importante, est relative à la législation des chutes d'eau et des réseaux de distribution d'énergie, Loi du 16 octobre 1919 et Circulaires ultérieures. (S. V.)

\section{$*$}

La Recherche méthodique des pannes d'automobiles, par R. Bardin, ingénieur électricien (E. S. M. E.), diplômé de l'Ecole Supérieure d'Aéronautique. In-16 broché de 46 pages, prix : 2 fr. $50 .-$ Desforges, Girardot \& $\mathrm{C}^{\circ}$, éditeurs, 27, quai des Grands-Augustins, Paris. En vente à la librairie Rey, Grande-Rue, à Grenoble.

Dans les voitures automobiles modernes, les accidents dus à la rupture de pièces mécaniques sont rares; mais par suite d'un déréglage ou d'un mauvais entretien, il peut arriver qu'un organe de la voiture, le moteur en particulier, vienne à mal fonctionner ou à refuser tout service.

Si certaines causes de mauvais fonctionnement sont apparentes et facilement réparables, il arrive par contre que des pannes dissimulées sont longues à trouver. Il y a lieu, dans ce cas, d'orienter les recherches avec esprit de méthode, d'éliminer successivement les causes de mauvais fonctionnement possibles afin de restreindre rapidement le champ des investigations. Une fois la cause trouvée, il est nécessaire d'y apporter remède et d'en éviter le retour.

C'est pour permettre à tout conducteur de se tirer d'affaire dans le cas de mauvais fonctionnement d'un organe de la machine, sans le secours d'un spécialiste, que sont traitées méthodiquement dans cet ouvrage les pannes de moteur, les pannes de transmission et de châssis, les pannes d'ćclairage et de démarrage électrique, les pannes de pneumatiques. 
Cet ouvrage s'adresse donc à tous les usagers de l'automobile et il leur sera indispensable sur la route où l'automobiliste est livré à lui-même, et ne doit souvent compter que sur ses propres ressources pour se lirer d'embarras. (S. V.).

Le problème actuel du condenseur à surface, par A. Delas. - Editions de la Revue Industrielle, 57, rue Pierre-Charron, Paris, 8 . Prix, dans ses bureaux : 5 francs.

L'évolution de la turbine à vapeur et la construction des grandes centrales modernes ont fait envisager sous un jour nouveau le problème du condenseur à surface, que, jusqu'ici, on s'était occupé de résoudre surtout par l'augmentation des surfaces d'échange. Il résulte de recherches récentes, qu'étant donné la surface d'échange et la circulation d'eau, la disposition des tubes à l'intérieur du condenseur joue un rôle important et qu'on peut même avoir intérêt, dans certaines conditions, à en diminuer notablement le nombre.

C'est ainsi que l'Union d'Electricité a été conduite, à sa centrale de Gennevilliers, à supprimer $26 \%$ des tubes de certains de ses condenseurs, et a obtenu, du fait de cette réduction, des résultats remarquables.

M. Delas, qui a étudié la question sous ce nouvel aspect, tant au point de vue théorique que pratique, en fait l'exposé dans cette brochure qui sera lue avec intérèt et profit par la totalité des ingénieurs et industriels qui ont à condenser de la vapeur.

Changes et Monnaies, par Louis Pommery. Préface de M. Albert Buisson, président du Conseil d'administration de la Banque nationale française du Commerce extérieur. Exposé de la crise monétaire universelle et de la situation monétaire des divers pays, au début de 1926 ; suivi d'un Manuel pratique des Changes a l'usage des industriels et des commerçants. Un volume in-8 de 600 pages, Giard, éditeur, Paris. En vente à la librairie Rey, Grande-Rue, à Grenoble, et à la Banque nationale française du Commerce extérieur (au siège social, 21, boulevard Haussmann, a Paris). Prix : 45 francs.

Cet ouvrage, qui vient à son heure, ne peut manquer d'intéresser tous ceux que préoccupe le sort du franc. Il sera d'un précieux secours aux industriels et aux commerçants qui font des affaires avec l'étranger, à l'intention spéciale desquels il a été, du reste, conçu et rédigé.

L'auteur a consacré un premier chapitre aux aspects théoriques et techniques du problème monétaire. Extrêmement nourrie. tout en restant toujours parfaitement claire, cette partie de l'ouvrage met au point à la lumière des événements des dernières années, toute une série de notions, trop souvent imprécises et cependant indispensables pour comprendre l'évolution de notre propre situation monétaire et de notre change.

La deuxième partie du livre - et la plus importante - se compose d'une suite de notices où $M$. Louis Pommery a réuni un ensemble absolument unique de renseignements sur la situation monétaire des divers pays au début de 1926 .

C'est là une véritable synthèse de l'histoire monétaire du monde depuis 1914 et une documentation inestimable, aussi bien pour l'homme d'affaires que pour l'économiste.

Tous ceux qui sont en relations d'affaires avec l'étranger : importateurs, exportateurs, financiers, capitalistes, et qui doivent savoir ce qu'est exactement le schilling autrichien, ou le pengoe hongrois, ou le zloty polonais, ou le tchervonetz, tous ceux que préoccupe le problème monétaire et qui veulent connaitre les méthodes grâce auxquelles les pays qui ont aujourd'hui triomphé cles troubles monétaires ont pu revaloriser ou stabiliser leur monnaie trouveront, dans ces chapitres du livre de $M$. Pommery, réponse aux multiples questions qu'ils se sont sans doute déjà posées.

Enfin, dans une troisième partie, M. Pommery a rassemblé tout ce qu'il faut savoir du marché des changes et des opérations qui s'y traitent. Il y a là quantité d'indications qui seront précieuses aux importateurs et aux exportateurs, tout particulièrement sur les opérations de change à terme et sur les méthodes de couvertures les plus propres à éviter les risques du change.

Dans le dernier chapitre, M. Pommery examine les clauses insérées dans les contrats en vue de se prémunir contre les dangers de la clépréciation monétaire, et précise pour chacume d'elles la position actuelle de la jurisprudence.
Ainsi conçu, ce livre essentiellement pratique, clair et toujours facile à lire malgré la technicité des sujets abordés, vient combler une lacune, à une heure où son intérêt n'a pu être souligné. Il est précédé d'une pénétrante préface de $M$. Albert Buisson, qu'il faut lire et méditer, car elle contient sur les difficiles problèmes de l'heure présente plus d'une leçon profonde. S'adressant aux producteurs, " aux hommes d'action qui travaillent avec une ardeur infatigable à la prospérité du pays ", M. Albert Buisson leur demande de s'instruire et de réfléchir sur les facteurs dont dépend la vie économique nationale : "Guidée par une raison éclairée, écrit-il, leur foi dans les destinées de notre pays n'en sera que plus robuste et plus efficace. Elle triomphera, car on peut dire, modifiant une formule célèbre, que pour entreprendre une tâche, il faut des raisons de persévérer et que, pour réussir, il faut posséder l'espoir raisonné du succès."

(S. V.).

Etablissement des bilans or, par MM. Raffegeau et Lacout. Un volume $16 \times 26$, de 150 pages. Payot, éditeur, 106, boulevard Saint-Germain, à Paris. En vente à la Librairie Rey, Grande Rue, à Grenoble. Prix : 15 francs.

Les auteurs de cet ouvrage, MM. P.-C. Raffegeau et A. Lacout, se sont attachés à décrire les conditions toutes particulières et encore trop peu connues en France - du financement des entreprises lorsque, l'unité monétaire s'avilissant, il devient indispensable d'accroître les fonds de roulement.

Comment fixer les prix de vente de manière à assurer en tout état de cause le renouvellement régulier des stocks ? Comment calculer l'amortissement des immeubles et du matériel ? Comment échapper à la dépréciation des éléments d'actifs exprimés en monnaie légale ? Comment apprécier l'importance réelle de bénéfices qui peuvent n'être qu'apparents ? Tels sont quelques-uns des problèmes vitaux, aussi bien pour la prospérité de chaque entreprise que pour l'avenir de l'économie nationale, qui ont été traités dans la seconde partie de l'ouvrage.

Plusieurs méthodes de correction des comptabilités tenues en monnaie-papier y sont clairement exposées.

Une dernière partie est consacrée à l'analyse des conditions dans lesquelles les entreprises allemandes ont dû, après la réforme monétaire de 1923, faire table rase de toute leur comptabilité passée et revenir à la pratique des bilans-or. Quelques statistiques instructives revèlent la perte de substance que la ruine monétaire et la désorganisation des compatabilités, qui en est la conséquence, ont infligée à l'économie allemande.

(S. V.)

Les changements apportés par la guerre à la richesse de la France (extrait du livre III du cours d'économie politique), par C. Colson, inspecteur général des Ponts-et-Chaussées, vice-président du Conseil d'Etat, membre de l'Académie des Scicnces morales et politiques. - Une brochure in-8 de 52 pages, 1926. Prix : $7 \mathrm{fr}$. Gauthier-Villars, éditeur. - En vente à la librairie Rey, GrandeRue, à Grenoble.

Le cours d'Economie politique professé par M. Colson, de 1892 à 1914, à l'Ecole des Ponts-et-Chaussées, puis, depuis 1918, partie à l'Ecole Polytechnique, partie à l'Ecole des Ponts-etChaussées, a été publié par l'auteur en six volumes in-8 d'environ 500 pages, dont la $2^{e}$ édition était épuisé au début de la guerre. Voulant résumer les enseignements du siècle de paix et de liberté compris entre la fin des guerres de la Révolution et de l'Empire et le début de la guerre mondiale; convaincu, d'autre part, que bien des années s'écouleraient avant qu'il fût possible de dégager les conséquences des bouleversements résultant de cette guerre, M. Colson fit paraître, de 1915 à 1919, une édition qu'il a appelée définitive, des quatre premiers volumes, contenant toutes les matières ordinaires d'un cours d'économie politique. Pour les volumes V (Finances publiques) et VI (Travaux publics et Transports) $)_{2}$ il s'est borné à joindre à un nouveau tirage du texte antérieur une annexe donnant les chiffres afférents à l'année 1913.

Depuis lors, un nouveau tirage a été fait des livres I (Théorie générale des phénomènes économiques) et II (le Travail et les Questions ouvrières). Leur texte n'a pas été modifié, mais des notes additionnelles y ont été ajoutées, peu nombreuses pour le premier, car les principes de la science n'ont pas été modifiés depuis sa date, assez importantes au contraire pour le livre II, traitant de matières dans lesquelles il était bon d'indiquer pas mal de faits nouveaux, avec leurs conséquences.

A son tour, le livre III (la propriété des biens corporels et incor- 
porels) va faire l'objet d'un nouveau tirage. Or, les chapitres III et IV de ce livre contiemnent une évaluation détaillée du total des revenus privés en France et de la fortune globale de la France en 1913, comparée avec les chiffres analogues en Angleterre, en Allemagne et aux Etats-Unis. Les domées statistiques manquent absolument pour mettre à jour ce travail et les évaluations en francs actuels changent, hélas ! trop rapidement pour garder longtemps quelque intérèt. Mais M. Colson a cru pouvoir tenter d'évaluer approximativement en francs-or, dans un chapitre $Y$, ajouté à ce volume, le cout de laguerre, les ressources qui y ont fait face. les revenus privés et la fortune globale de la France en 192 $4-25$. La traduction de ces chiffres en francs-papier aux divers cours du change pourra varier largement, mais les réparations étant très avancées, les emprunts de guerre et d'après-guerre terminés, les conditions de règlement des dettes internationales à peu près arrètées, on peut penser que les conséquences de la guerre en valeur peuvent être aujourd'hui mesurées et faire l'objet d'une estimation sérieuse.

En attendant le nouveau texte complet du livre III, MN. Gauthier-Villars ont publié un tirage à part de ce chapitre additionnel. qui contient l'indication, aussi précise que possible, des conséquences de la guerre pour la richesse de la France.

(S. T.).

\section{$\star^{\star} \star \star$}

Les Réserves d'Energie, par Rigaud, ingénieur des mines, préface de M. Lecornu, nembre de l'Institut. Un volume $19 \times 13,5$ : de 295 pages. Encyclopédie, Léauté. - Gauthier-Villard \& $\mathrm{C}^{\circ}$. Masson \& $\mathrm{C}$, éditeurs. - En vente à la librairie Rey, GrandeRue, à Grenoble.

Parmi les questions intéressant les destinées de l'humanité, aucune dans le domaine matériel ne surpasse en importance celle de la quantité totale d'énergie utilisable. Il est vrai que paraît encore bien lointaine l'époque où l'homme, suivant la conclusion finale de M. Rigaud, se verra, par l'épuisement des réserves d'énergie, contraint d'abandonner toutes les conquêtes de l'industrie pour se contenter, comme nos premiers ancêtres de la vie purement agricole. Ainsi que l'a fait remarquer M. de Chaumont dans La Science Moderne ", vents, marées, mascarets, soleil, substances radioactives, ne sont présentement qu'une poussière d'énergie. Leurs différentes applications ne sont guère que des curiosités. Comme le dit M. Lecornu, dans la préface de cet excellent ouvrage : Nul doute que le jour où la nécessité se fera plus impérieuse, des inventeurs de génie ne trouvent le moyen de rendre plus accessibles ces énergies indociles. On sera probablement conduit à développer dans d'énormes proportions tous les modes de transport de force et d'accumulation.

On fera de plus en plus appel à la chaleur centrale de notre globe. Peut-être réussira-t-on à mieux capter l'énergie solaire par la culture intensive de végétaux appropriés. D’une façon générale, les circonstances conduiront à exécuter bien des projets que le chiffre de la dépense rendrait pour l'instant irréalisables.

Si l'on songe, d'après Le Bon, que dans une pièce de 5 centimes, il $\mathrm{y}$ a (énergie atomique) 510 milliards de kilogrammètres emmagasinés... L'énergie de chaque atome se trouve en quelque sorte emprisonnée dans une enveloppe imperméable qui la maintient à l'état potentiel ; à peine certains atomes, ceux du radium notamment, la laissent-ils filtrer au compte-gouttes. Si l'on trouvait le moyen de forcer le débit, non seulement pour le radium, mais aussi pour les substances moins rares, il n'y aurait plus besoin de chercher autre chose.

Taut cela est le secret de l'avenir et quant au présent, il faut reconnaitre que le stock d'énergies pratiquement disponibles s'appauvrit avec une rapidité inquiétante. L'auteur, après avoir passé en revue toutes les formes d'énergies connues, conclut qu'il faut éviter tout gaspillage d'énergie.

Cet ouvrage, où l'auteur, appuyé sur une abondante documentation, examine le problème de l'énergie sous toutes ses faces, sera lu avec beaucoup d'intérêt.

(S. V.).

\section{$\star_{\star \star \star *}^{*}$}

Les Problèmes de la route. - Numéro spécial, édité, à l'occasion du Congrès de la Route de Milan, par la Section industrielle du Groupement pour le Commerce et l'Industrie. - Un vol. de 170 pages, 80 fig. Prix : $15 \mathrm{fr}$.

Voici les principales études traitées dans cet ouvrage : Le Problème routier en France (Le Gavrian). - Le Problème comparé des routes en France et en Amérique (Antoine). - Les routes coloniales (Teissier). - Les problèmes de la voirie municipale (Boutteville).
Considérations générales sur les routes en béton (Mesnager). - Considérations techniques sur les routes en béton (Berenguier). La préparation du liant bitumineux (Laxel), - - Les revètements asphaltiques (Champilou). - Le tarmacadam (Roll). - Le goudronnage superficiel (Lassailly). -.... Les chaussées silicatées (13edier). .. - Essais des matériaux pour les roules (Anstett).... Lád propreté des routes et les liants bitumineux (Mascart).

Les industriels et la route (Mascot et Leroux).

Des crises économiques, commerciales et financières, par $\Lambda$.

Poirier, directeur à la Compagnie des organisateurs-complables, éditions de "La Comptabilité et les Affaires ", 22, rue de l'Arcade, à Paris. - En vente chez Edmond Langlois \& $C^{\circ}$, 186, faubourg Saint-Martin, Paris, et a la librairie Rey, GrandeRue, à Grenoble. - Prix : 10 franes.

La caractéristique essentielle de la situation présente est l'instabilité monétaire. Ses origines ont été souvent décrites, ses conséquences sont sensibles à tous ceux qui, dans leur patrimoine, dans leurs affaires, dans les occupations mèmes de leur existence, subissent le contre-coup de l'appauvrissement national. Cependant l'opinion publicue "le monde des affaires ". restent insuffisanment renseignés à ce sujet, malgré qu'une littérature abondante et variée sur les questions économiques et financières, soit mise a leur disposition.

Aussi, sommes-nous persuadés que la brochure édité trís opportunément par la Revue technique "I a Comptabilité ct les Affaires "intéressera vivement tous ceux - ils sont, hélas ! trìs nombreux - qui souffrent, à des degrés divers, du déséquilibre économique actuel.

M. Poirier, auteur de ce travail très condensé, a voulu montrer à ses lecteurs l'intérêt que présente l'étude pratique des crises, l'importance pour le milieu des affaires, de leur prévision. Dans ce but, et dans un stỵle très simple, se succèdent les dix chapitres suivants :

I. - Qu'est-ce qu'une crise ?

II. - La réponse des économistes.

III. - Du rythme de la vie économique.

IV. - Du ròle de l'épargne dans les crises générales de surproduction.

V. - Il n'y a pas "une " cause des crises, mais "des " causes. VI. - Caractère de périodicité des crises.

VII. - Exemple de crise "d'après-guerre" 1920 (Japon, EtatsUnis, France).

VIII. - Pour faire le point... Etat de la situation économique en France.

IX. - De la "prévision" des crises (Etats-Unis, Angleterre, France).

X. - Comment assurer la marche des entreprises en période de crises.

Ajoutons, pour ètre complet, que la brochure contient en annexes en plus d'une bibliographie des principaux ouvrages à consulter sur la question, un certain nombre de tableaux et huit graphiques en deux couleurs, illustrant le texte très clair de ce travail consciencieux auquel nous souhaitons un vif succès. (S. V.).

\section{$\star^{* * *}$}

Pour le Relieur (amateur ou professionnel), par Ch. Roux. Un volume de $19 \times 12$, de 161 pages, Dunod, éditeur, 92, rue Bonaparte, Paris. - En vente : librairie Rey, Grande-Rue, à Grenoble, broché, 12 fr.

Ce petit ouvrage est des plus intéressants. Il donne, pour les amateurs, quantité de recettes que ceux-ci ne pourraient trouver dans les livres ordinaires. Les artisans pourront y puiser aussi d'utiles renseignements : travail du dos, débrochage, battage, couture, pressage, travail de la tranche, rognage, coloration, tranchefile ; dorure, apprêt, pommades, palette à combinaison; marbrures, reliures spéciales, brochures ordinaires et de fantaisie; colles et collages ; entoilage; on y trouvera en particulier la manière d'exécuter une reliure ordinaire avec un matériel coûtant à peine une vingtaine de franes, le système anglais de reliure pour bibliothèques publiques, d'une solidité défiant les traitements les plus rudes, système dont on chercherait en vain la description dans tous les manuels de reliure publiés en français. L'auteur fait aussi une large place aux systemes de brochage fantaisie et de reliures démontables pour fiches qui sont maintenant si pratiques.

Le Gerant: P LEGENDRE

Anc. Etabts Logendre, 14, rue Bellecordière, Lyon. - j. Bataillard, Directeur 